Artículo científico

Volumen 31(2):341-351. Mayo-agosto, 2020

e-ISSN 2215-3608, doi:10.15517/am.v31i2.37806

http://www.revistas.ucr.ac.cr/index.php/agromeso

\title{
Productividad de vacas lecheras suplementadas con ensilaje de haba alpargata o remolacha forrajera ${ }^{1}$
}

\section{Productivity of dairy cows supplemented with silage of fava bean or fodder beet}

\author{
David Felipe Nieto-Sierra ${ }^{2}$,Elizabeth Lagos-Burbano ${ }^{2}$, Yesid Avellaneda-Avellaneda ${ }^{2}$ Edwin Castro-Rincón ${ }^{2}$
}

1 Recepción: 27 de junio, 2019. Aceptación: 11 de noviembre, 2019. Este trabajo formó parte del proyecto de tesis de Maestría del primer autor, llevado a cabo en el marco del proyecto denominado "Mejoramiento de la oferta forrajera, optimización de los sistema de alimentación y aseguramiento de la calidad e inocuidad de la leche en el trópico alto del departamento de Nariño", financiado por el Sistema General de Regalías y Gobernación de Nariño, ejecutado por el Centro de Investigación Obonuco de la Corporación Colombiana de Investigación Agropecuaria (AGROSAVIA), Pasto, Nariño.

2 Corporación Colombiana de Investigación Agropecuaria (AGROSAVIA). Centro de Investigación Obonuco. Km 5, Vía Pasto, Obonuco, Nariño, Colombia.dnieto@agrosavia.co (https://orcid.org/0000-0003-4808-1201), elagos@agrosavia.co (https://orcid.org/0000-0002-50989908), yavellaneda@agrosavia.co (https://orcid.org/0000-0003-2471-5863), ecastro@agrosavia.co (https://orcid.org/0000-0001-9841-8242).

\section{Resumen}

Introducción. Los sistemas de producción de leche son afectados por el cambio climático, presentan estacionalidad en la producción forrajera, y sumado a esto, predominan razas con altas exigencias nutricionales, lo que conlleva a que los productores acudan permanentemente al uso de concentrados que incrementan los costos de producción. Objetivo. El objetivo del presente trabajo fue evaluar la producción y calidad de la leche en vacas suplementadas con ensilaje de haba alpargata (Vicia faba L.) y remolacha forrajera (Beta vulgaris L.). Materiales y métodos. El estudio se realizó entre julio y agosto del 2018 en el Centro de Investigación Obonuco de AGROSAVIA, municipio de Pasto, Nariño, Colombia. Se emplearon doce vacas de la raza F1 (Kiwi Cross X Holstein), de primer parto (93 días de lactancia), con un peso vivo promedio de $460 \mathrm{~kg}$. Fueron mantenidas en potreros con gramíneas, leguminosas y agua ad libitum. Durante tres periodos de quince días cada uno, se evaluaron tres niveles de inclusión de ensilaje $\left(0,4\right.$ y $\left.8 \mathrm{~kg} \mathrm{día}^{-1}\right)$ de haba y remolacha, mediante un diseño experimental cuadrado latino $3 \times 3$ para cada especie. Se registró la producción diaria por animal y se determinó la calidad composicional de la leche (grasa, proteína, sólidos totales y nitrógeno ureico en leche MUN). Resultados. Con el mayor nivel de inclusión (8 kg vaca $^{-1}$ día $\left.^{-1}\right)$, el ensilaje de haba alpargata tuvo efecto $(\mathrm{p}<0,05)$ en el porcentaje de proteína, mientras que, el ensilaje de remolacha forrajera influyó $(\mathrm{p}<0,05)$ en el porcentaje de grasa. Ambas especies no influyeron en la producción, sólidos totales y MUN. Conclusión. El suministro de ensilajes de haba y remolacha tuvieron efecto en la calidad composicional de la leche, siendo alternativas alimenticias en los sistemas de producción de leche del trópico alto de Nariño, que disminuyen la estacionalidad y el reemplazo de fuentes comunes.

Palabras claves: grasa, proteína, lactancia, cultivos forrajeros. 


\begin{abstract}
Introduction. Milk production systems are affected by climate change, presenting seasonality in forage production, and added to this, breeds with high nutritional requirements predominate, which leads producers to permanently use concentrates that increase the costs of production. Objective. The objective of this work was to evaluate milk production and quality in cows supplemented with espadrille bean (Vicia faba L.) and fodder beet (Beta vulgaris L.) silage. Materials and methods. The study was conducted at the Obonuco Research Center of AGROSAVIA, municipality of Pasto, Nariño, Colombia. Twelve cows of the F1 breed (Kiwi Cross X Holstein) were used, of first childbirth (93 days of breastfeeding), with an average live weight of $460 \mathrm{~kg}$. Cows were kept in paddocks with grasses, legumes, and water ad libitum. For three periods of fifteen days each, three levels of silage inclusion $\left(0,4\right.$ and $\left.8 \mathrm{~kg} \mathrm{day}^{-1}\right)$ of bean and beet were evaluated, using a 3x3 Latin square experimental design for each species. Daily production of each animal was recorded and the compositional quality of the milk (fat, protein, total solids and milk urea nitrogen MUN) was determined. Results. With the highest level of inclusion $\left(8 \mathrm{~kg} \mathrm{cow}^{-1} \mathrm{day}^{-1}\right)$, the alpargata bean silage had an effect $(\mathrm{p}<0.05)$ on the protein percentage, while the fodder beet silage influenced $(\mathrm{p}<0,05)$ in the percentage of fat. Both species did not influence production, total solids, and MUN. Conclusion. The supply of bean and beet silage influenced the compositional quality of milk, providing food alternatives in the milk production systems of the upper tropics of Nariño, allowing seasonality and the replacement of common sources to be reduced.
\end{abstract}

Keywords: fat, protein, lactation, forage crops.

\title{
Introducción
}

En el ámbito latinoamericano, la producción de ganado bovino tradicionalmente ha sido una de las principales actividades productivas del sector agrícola, lo cual obedece en gran parte, a la presencia de amplias sabanas y bosques con que cuenta la región. Sin embargo, la ganadería de los trópicos latinoamericanos enfrenta problemas relacionados con la cantidad, calidad y productividad de las pasturas, en particular durante los prolongados períodos de déficit hídrico. Este es un problema a gran escala y se debe a la elevada fracción de la base forrajera disponible que está conformada por pasturas nativas de baja productividad, y por especies introducidas sin adecuada adaptación. En la ganadería tropical coexisten múltiples sistemas de producción en diferentes pisos térmicos, distintos grados de intensificación y ubicados en ambientes socioeconómicos de muy diversa naturaleza (Holmann et al., 2003).

Los sistemas especializados de producción de leche en Colombia se desarrollan básicamente bajo condiciones de trópico de altura (1800 a $3000 \mathrm{msnm}$ ) y precipitaciones anuales entre 1600 a $2200 \mathrm{~mm}$ (Morales et al., 2013). La alimentación está constituida principalmente de una base forrajera de monocultivos de kikuyo (Cenchrus clandestinum), especie que predomina en la mayoría de los sistemas de producción de leche en las microrregiones de altiplanicies y laderas frías, sin embargo, presenta baja producción por inadecuadas prácticas de manejo, sobrepastoreo y por su susceptibilidad a heladas y sequías (Echeverry et al., 2010; Morales et al., 2013; Carulla y Ortega, 2016).

La producción de forrajes es estacional, con pastos abundantes en épocas de lluvia y bajos rendimientos en épocas de mínima precipitación, lo que trae como consecuencia pérdidas marcadas en la producción animal (Arteaga, 2009; Betancourt et al., 2012). Esto conlleva a que los productores acudan permanentemente al uso de alimentos concentrados que incrementan los costos de producción (Molano et al., 2011). Por esta razón, es importante explorar alternativas alimenticias como cultivos forrajeros y arbóreos de origen regional, que contribuyan a mejorar la oferta de alimento de buena calidad a un menor valor económico (Trujillo, 2009). 
Actualmente se han estudiado diferentes propuestas para diversificar la alimentación de los bovinos en sistemas de producción, las cuales promueven la implementación de cultivos forrajeros, donde se destaca el haba (Vicia faba L.) y la remolacha forrajera (Beta vulgaris L.), las cuales pueden ser utilizadas en sistemas de corte y acarreo o para la elaboración de ensilajes, debido a que presentan gran producción de biomasa y buena adaptación (Sañudo et al., 1999; Anderson et al., 2015).

El cultivo del haba es de gran importancia dentro en la actividad agrícola en las zonas frías del país, considerado como un cultivo de rotación en regiones con alturas superiores a los $2800 \mathrm{msnm}$. Se calcula que en Colombia se dedican aproximadamente 5000 ha año ${ }^{-1}$ a este cultivo, cuya producción se consume, $60 \%$ grano verde y $40 \%$ en grano seco. Esta leguminosa es una valiosa fuente alimenticia, porque presenta entre el 26 y $35 \%$ de proteína (Checa, 1994). El haba tiene un buen comportamiento entre 2700 y $3200 \mathrm{msnm}$; sin embargo, su mejor capacidad productiva se logra entre los 2900 y 3100 msnm (Sañudo et al., 1999).

El haba forrajera (Vicia faba L.) es una leguminosa fijadora del nitrógeno que mejora la composición del suelo y es un cultivo anual muy adecuado para la rotación corta de cultivos cuando se usa como ensilado (Borreani et al., 2009). Tanto en monocultivo como asociada con otras especies, representa una buena alternativa alimenticia, ya que el suministro de ensilaje de esta especie mejora la producción en proteína y energía, afectando positivamente la calidad de leche y reduciendo los costos de producción (Baizán et al., 2015).

El potencial de la remolacha radica en su rendimiento en suelos salinos, donde pocos cultivos crecen, ya que su raíz toma agua de lo más profundo en el perfil del suelo, bajando el nivel freático y ejerciendo efecto de biorremediación (Anderson et al., 2015). La remolacha es un cultivo suplementario, con un elevado contenido energético que puede estimular la producción de leche, debido a su palatabilidad y digestibilidad; además, complementa adecuadamente la calidad de la dieta de los animales alimentados a base de forrajes y ofrece el potencial para reemplazar el grano en las raciones de vacas lecheras, proporcionan ventajas económicas. El principal inconveniente de la remolacha es su bajo contenido de materia seca (MS), que representa una limitante para su elevado uso en las raciones (Lafaux et al., 2015; Evans et al., 2016).

La remolacha forrajera posee un alto contenido de azúcares, tiene una alta densidad de energía, apropiada para las necesidades de los animales de alto rendimiento productivo, permitiendo una óptima fermentación ruminal. La suplementación de energía que proporciona este cultivo a los rumiantes, mejora los contenidos de proteína y grasa en leche, debido a que los microorganismos del rumen son favorecidos por el contenido de azúcares, lo que hace que la fibra sea mejor fermentada. Estos azúcares también favorecen la síntesis microbial de proteína en el rumen, lo que influye en la producción de caseína (proteína de la leche) (Rodríguez et al., 2007).

El objetivo de este trabajo fue evaluar la producción y calidad de la leche de vacas suplementadas con ensilaje de haba alpargata (Vicia faba L.) y remolacha forrajera (Beta vulgaris L.).

\section{Materiales y métodos}

\section{Localización}

El estudio se realizó durante los meses de julio y agosto de 2018 en el hato lechero del Centro de Investigación Obonuco de la Corporación Colombiana de Investigación Agropecuaria (AGROSAVIA), municipio de Pasto, Nariño, Colombia, ubicado a una altura de $2905 \mathrm{msnm}, 01^{\circ} 11^{\prime} 28,3^{\prime} \mathrm{N}, 77^{\circ} 19^{\prime} 08,8^{\prime \prime} \mathrm{W}$, temperatura promedio anual de $12{ }^{\circ} \mathrm{C}$ y con una precipitación pluvial promedio anual de $950 \mathrm{~mm}$, datos obtenidos de la estación meteorológica (Vintage station pro 2), ubicada en el CI Obonuco, AGROSAVIA, en 2018. 


\section{Animales y manejo}

Se emplearon doce vacas F1 (Kiwi Cross x Holstein) de primer parto, con un promedio de 93 días en lactancia y una producción de $131 \mathrm{vaca}^{-1}$ día $^{-1}$, con un peso vivo promedio de $460 \mathrm{~kg}$. El consumo de agua fue ad libitum, se mantuvieron en potreros con predominio de gramíneas kikuyo (Cenchrus clandestinus) y ryegrass, de las variedades Samson, Sterling, Ohao y One 50 (Lolium multiflorum), y tréboles (Trifolium sp.) como representantes de las leguminosas, además se observó en las praderas falsa poa (Holcus lanatus) y azul orchoro (Dactylis glomerata). Se realizó un pastoreo rotacional con cerca eléctrica, ajustando diariamente el área de pastoreo para asegurar una oferta teórica de materia seca por animal de 3,0 kg MS $100 \mathrm{~kg}^{-1}$ de peso vivo. Las desparasitaciones internas se realizaron con productos a base de Fenbendazol, con intervalos de tres meses acompañadas con la aplicación de complejos multivitamínicos (vitaminas A, D y E). Para el control de la mosca se aplicó un antiparasitario externo (Ethion), el tiempo de administración varió dependiendo de la presencia de este insecto.

\section{Tratamientos}

Se establecieron tres niveles de inclusión de ensilaje $\left(0,4\right.$ y $8 \mathrm{~kg}_{\text {de ensilaje vaca }}{ }^{-1}$ día $\left.^{-1}\right)$ para cada especie, haba alpargata (Vicia faba L.) y remolacha forrajera (Beta vulgaris L.). La suplementación se realizó en tres periodos de 15 días, para un total de 45 días, donde los primeros siete días correspondieron al periodo de acostumbramiento y los ocho días siguientes fueron utilizados como período de medición (Jefery, 1970; Stobbs y Sandland, 1971; Elgersma et al., 2004). El suministro se realizó en dos momentos, en el ordeño de la mañana (4:00) y de la tarde (15:00).

\section{Composición química}

En el Cuadro 1 se presenta la composición nutricional de la mezcla de las gramíneas y de los ensilajes empleados, los cuales fueron obtenidos mediante la técnica de espectroscopia de reflectancia en infrarrojo cercano NIRS (NIRS DS 2500 - FOSS Analytical A/S - Dinamarca) (Ariza et al., 2017), con base en los métodos de referencia: proteína cruda (PC) (AOAC984.13), fibra en detergente neutro (FDN) (AOAC2002.04), fibra en detergente ácido (FDA) (AOAC973.18), lignina (LIG) (se determinó a partir del residuo insoluble del detergente ácido tratado con ácido sulfúrico $12 \mathrm{M}$ ), hemicelulosa (HEM) (diferencia entre FDA y FDN), digestibilidad (DIG) y energía neta de lactancia (ENL) (mediante ecuaciones elaboradas por el laboratorio).

Cuadro 1. Variables nutricionales de ensilaje de haba alpargata (Vicia faba L.) y remolacha forrajera (Beta vulgaris L.) en el Centro de Investigación Obonuco de la Corporación Colombiana de Investigación Agropecuaria (AGROSAVIA), municipio de Pasto. Colombia. 2018.

Table 1. Nutritional variables of espadrille bean (Vicia faba L.) and fodder beet (Beta vulgaris L.) silage at Obonuco Research Center of the Colombian Agricultural Research Corporation (AGROSAVIA), municipality of Pasto. Colombia. 2018.

\begin{tabular}{lcccccccc}
\hline \multirow{2}{*}{ Especie } & MS & PC & FDN & FDA & LIG & HEM & DIG & ENL \\
\cline { 2 - 9 } & & & & \% & & & & $\left(\mathbf{M c a l ~ k g}^{-1} \mathbf{M S}\right)$ \\
\hline Gramíneas & 21,57 & 19,13 & 49.10 & 23,78 & 6,09 & 25.31 & 67,28 & 1,39 \\
Haba alpargata & 24,07 & 13,52 & 40,02 & 7,04 & 3,50 & 32,98 & 68,07 & 1,41 \\
Remolacha forrajera & 14,75 & 17,69 & 38,67 & 5,99 & 6,43 & 32,69 & 71,72 & 1,49 \\
\hline
\end{tabular}

MS: materia seca, PC: proteína cruda, FDN: fibra en detergente neutro, FDA: fibra en detergente ácido, LIG: lignina, HEM: hemicelulosa, DIG: digestibilidad de la materia seca, ENL: energía neta de lactancia / MS: dry matter, PC: crude protein, FDN: fiber in neutral detergent, FDA: fiber in acid detergent, LIG: lignin, HEM: hemicellulose, DIG: dry matter digestibility, ENL: lactation net energy. 


\section{Variables medidas}

Se registró la producción diaria de leche de cada animal y se tomaron muestras de $100 \mathrm{ml}$ para evaluar la calidad composicional los días 13, 14 y 15 de cada periodo. Se determinó la concentración de grasa, proteína, sólidos totales y nitrógeno ureico en leche (MUN) mediante el método de espectroscopia infrarroja (AOAC, 2005). Las muestras se procesaron empleando el equipo FOSS MilkoscanTM 7 RM - FOSS Analytical A/S - Dinamarca, en el laboratorio de leche del C.I Obonuco de AGROSAVIA, Pasto, Colombia.

\section{Diseño experimental y análisis estadístico}

Se empleó un diseño experimental cuadrado latino 3x3 para cada especie utilizada como suplemento (Martínez et al., 2011), con tres periodos de quince días, para un tiempo total de evaluación de 45 días, la unidad experimental correspondió a una vaca en lactancia.

Modelo matemático (Martínez et al., 2011).

$Y_{i j(k)}=\mu+F_{i}+C_{j}+T_{(k)}+E_{i j(k)}$

Donde:

$Y_{i j(k)}=$ Observación de la unidad experimental.

$\mu=$ media general.

$F_{i}=$ efecto del periodo $i$.

$C_{j}=$ efecto del animal $j$.

$T_{(k)}=$ efecto del tratamiento $k$.

$E_{i j(k)}=$ error experimental de la u.e $i, j$.

\section{Resultados}

En los materiales usados para la evaluación en animales se determinó la calidad nutricional, observando que el mejor contenido de proteína se obtuvo en la pastura $(19,13 \%)$, considerada de buena calidad, seguida por la remolacha forrajera $(17,69 \%)$ y el haba forrajera $(13,52 \%)$. En cuanto a materia seca, se evidenció que materiales como la remolacha forrajera se encontraron por debajo de lo óptimo para un proceso de ensilaje $(<15 \%)$, frente al haba y la pastura que estuvieron en rangos adecuados ( 20 a $24 \%$ ). Los contenidos de energía observados estuvieron en los rangos adecuados para la alimentación de vacas de leche en la zona en estudio (1,39-1,49 Mcal kg MS $\left.{ }^{-1}\right)$ (Cuadro 1).

El suministro de ensilaje de haba alpargata influyó $(\mathrm{p}<0,05)$ en el porcentaje de proteína de la leche, mientras que el ensilaje de remolacha forrajera influyó $(\mathrm{p}<0,05)$ en el porcentaje de grasa, con el mayor nivel de inclusión para ambas especies. La producción láctea, sólidos totales y nitrógeno ureico en leche no se vieron afectadas con la inclusión de los diferentes niveles de ensilaje de haba o remolacha en la dieta de los animales evaluados (Cuadros 2 y 3 ).

Las vacas suplementadas con ensilaje de remolacha forrajera produjeron mayor cantidad de litros por día $\left(14,18\right.$ 1), con mayor contenido de grasa $(4,10 \%)$ y nitrógeno ureico en la leche (MUN) $\left(16,61 \mathrm{mg} \mathrm{dl}^{-1}\right)$; por el contrario, las vacas suplementadas con ensilaje de haba alpargata presentaron mayor contenido de proteína (3,56 $\%)$ y sólidos totales en leche $(13,06 \%)$. 
Cuadro 2. Producción y composicional de leche de vacas suplementadas con ensilaje fresco de haba alpargata (Vicia faba L.), en el hato lechero del Centro de Investigación Obonuco de la Corporación Colombiana de Investigación Agropecuaria (AGROSAVIA), municipio de Pasto. Colombia. 2018.

Table 2. Production and composition of milk from cows supplemented with fresh espadrille bean (Vicia faba L.) silage, in the dairy herd of the Obonuco Research Center of the Colombian Agricultural Research Corporation (AGROSAVIA), municipality of Pasto. Colombia. 2018.

\begin{tabular}{|c|c|c|c|c|c|}
\hline $\begin{array}{c}\text { Tratamiento } \\
\left(\text { kg ensilaje vaca }^{-1} \text { día }^{-1}\right)\end{array}$ & $\begin{array}{c}\text { Leche } \\
\left(\text { l día }^{-1}\right)\end{array}$ & Grasa & $\begin{array}{c}\text { Proteína } \\
(\%)\end{array}$ & Sólidos & $\begin{array}{c}\text { MUN } \\
\left(\mathrm{mg} \mathrm{dl}^{-1}\right)\end{array}$ \\
\hline 0 & $13,61^{\mathrm{a}}$ & $4,01^{\mathrm{a}}$ & $3,56^{\mathrm{ab}}$ & $13,00^{\mathrm{a}}$ & $16,21^{\mathrm{a}}$ \\
\hline 4 & $13,27^{\mathrm{a}}$ & $4,08^{\mathrm{a}}$ & $3,50^{\mathrm{b}}$ & $13,07^{\mathrm{a}}$ & $16,93^{\mathrm{a}}$ \\
\hline 8 & $13,58^{\mathrm{a}}$ & $4,04^{\mathrm{a}}$ & $3,61^{\mathrm{a}}$ & $13,12^{\mathrm{a}}$ & $16,15^{\mathrm{a}}$ \\
\hline Media & 13,49 & 4,04 & 3,56 & 13,06 & 16,43 \\
\hline EEM & 2,69 & 0,02 & 0,01 & 0,07 & 1,71 \\
\hline
\end{tabular}

a-b-c Letras diferentes entre columnas indican diferencia estadística significativa $(p<0,05)$. MUN: nitrogeno ureico en leche. EEM: error estandar de la media / ${ }^{\mathrm{a}-\mathrm{b}-\mathrm{c}}$ Different letters between columns indicate significant statistical difference $(\mathrm{p}<0.05)$. MUN: milk ureic nitrogen. EEM: standard error mean.

Cuadro 3. Producción y composicional de leche de vacas suplementadas con ensilaje fresco de remolacha forrajera (Beta vulgaris L.), en el hato lechero del Centro de Investigación Obonuco de la Corporación Colombiana de Investigación Agropecuaria (AGROSAVIA), municipio de Pasto. Colombia. 2018.

Table 3. Production and composition of milk from cows supplemented with fresh fodder beet (Beta vulgaris L.) silage, in the dairy herd of the Obonuco Research Center of the Colombian Agricultural Research Corporation (AGROSAVIA), municipality of Pasto. Colombia. 2018.

\begin{tabular}{cccccc}
\hline $\begin{array}{c}\text { Tratamiento }(\mathbf{k g} \\
\left.\text { ensilaje vaca }^{-1} \mathbf{d i ́ a}^{-1}\right)\end{array}$ & $\begin{array}{c}\text { Leche } \\
\left(\mathbf{l ~ d i ́ a ~}^{-1}\right)\end{array}$ & Grasa & $\begin{array}{c}\text { Proteína } \\
(\%)\end{array}$ & Solidos & $\begin{array}{c}\text { MUN } \\
\left(\mathbf{m g ~ d l}^{-1}\right)\end{array}$ \\
\hline 0 & $14,44^{\mathrm{a}}$ & $4,06^{\mathrm{b}}$ & $3,47^{\mathrm{a}}$ & $13,00^{\mathrm{a}}$ & $16,57^{\mathrm{a}}$ \\
4 & $14,13^{\mathrm{a}}$ & $3,96^{\mathrm{b}}$ & $3,51^{\mathrm{a}}$ & $12,94^{\mathrm{a}}$ & $16,37^{\mathrm{a}}$ \\
8 & $13,97^{\mathrm{a}}$ & $4,26^{\mathrm{a}}$ & $3,44^{\mathrm{a}}$ & $13,15^{\mathrm{a}}$ & $16,88^{\mathrm{a}}$ \\
Media & 14,18 & 4,10 & 3,47 & 13,03 & 16,61 \\
EEM & 2,90 & 0,02 & 0,01 & 0,07 & 2,75 \\
\hline
\end{tabular}

${ }^{a-b-c}$ Letras diferentes entre columnas indican diferencia estadística significativa $(p<0,05)$. MUN: nitrógeno ureico en leche. EEM: error estándar de la media / ${ }^{\mathrm{a}-\mathrm{b}-\mathrm{c}}$ Different letters between columns indicate significant statistical difference $(\mathrm{p}<0.05)$. MUN: milk ureic nitrogen. EEM: standard error mean.

\section{Discusión}

Los trabajos que han evaluado la inclusión de haba en sistemas de alimentación de rumiantes han trabajado con el haba en grano. Se evaluó la inclusión de $10 \%$ de hojuelas de haba (cocinadas y secadas previamente) en concentrados para la alimentación de vacas de leche de la raza Holstein como reemplazo de proteína proveniente de soya, sin obtener diferencias significativas en la producción de leche, grasa, proteína y sólidos totales, similar a 
lo encontrado en este estudio (Volpelli et al., 2010; 2012), a excepción del porcentaje de proteína en leche el cual sí presentó diferencias, probablemente asociado al contenido de proteína en el material de haba empleado (13,52 $\%$ ), siendo mayor al máximo nivel de inclusión (8 $\mathrm{kg}_{\text {ensilaje vaca }}^{-1}$ día $\left.^{-1}\right)$. Por otro lado, Puhakka et al. (2016) y Volpelli et al. (2009) reportan un mayor contenido de urea en la leche de vacas que recibieron dietas con haba, debido al aumento del amoniaco en el rumen, asociado a la alta degradabilidad de las proteínas de esta fuente, resultados que contrastan con los obtenidos en este trabajo y que pueden estar relacionados con el alto consumo de proteína proveniente del forraje en los animales que no consumieron ensilaje de haba.

Al evaluar la inclusión de $10 \%$ de haba y $10 \%$ de arveja (Pisum sativum) en rumiantes productores de leche, se obtuvo una reducción en la producción, pero un aumento en el contenido de grasa (Mordenti et al., 2007) que, al compararlo con lo obtenido en este estudio para los tres niveles de inclusión, la dieta de haba + arveja presentó una mayor producción de leche $(31,841)$ pero un menor porcentaje de grasa $(2,66 \%)$. Cabe resaltar que en este estudio se empleó la raza Kiwi Cross, la cual se caracteriza por su buena composición de leche, pero su producción es menor que la de la raza Holstein (Montgomerie, 2005; Rowarth, 2013) y se suministró ensilaje de haba (planta completa: tallos, hojas, vainas) a diferencia de los anteriores trabajos donde se suministró únicamente el grano.

Con la inclusión de 1,7 $\mathrm{kg} \mathrm{día}^{-1}$ de frijol verde de haba por animal se encontró una producción de 20,48 1, con $4,9 \%$ de grasa y $3,6 \%$ de proteína (Osmane et al., 2017), resultados superiores a los observados en los tres niveles de inclusión de ensilaje de haba del presente estudio, excepto para la proteína, la cual fue similar y en ambos estudios estuvo afectada por la inclusión de haba en la dieta.

En el presente trabajo se observó un mayor contenido de proteína al obtenido por Argamentería et al. (2005), quienes evaluaron el ensilaje de haba en asocio con triticale en estado de vainas con grano, y grano lechoso respectivamente, donde obtuvieron promedios de producción de leche, contenido de grasa y proteína de 161 día $^{-1}, 4,44 \%$ y 3,03\%, respectivamente. Al evaluar el ensilaje de haba en asocio con colza (Brassica napus), se obtuvo mayor producción de leche, pero menores porcentajes de grasa y proteína $(28,421,3,97 \%, 3,37 \%$, respectivamente) (Benaouda et al., 2013). Gran parte de esta diferencia se asocia con el tipo de raza, en los trabajos de Argamentería et al. (2005) y Benaouda et al. (2013) se emplearon vacas de raza Holstein y en este estudio se utilizaron animales F1 (Holstein x Kiwi Cross) (Montgomerie, 2005; Rowarth, 2013).

Los resultados obtenidos para el cultivo de haba, indican que su inclusión en la dieta de vacas lecheras se constituye como una buena alternativa, por su alto contenido de proteína y bajo contenido de FDN (15,97\% y $55,12 \%$, respectivamente), además, presenta un valor energético equivalente a las gramíneas (Martínez et al., 2017); sin embargo, Mordenti et al. (2007) y Puhakka et al. (2016) afirmaron que la incorporación de haba en la dieta reduce la ingesta de materia seca y la producción de leche en vacas de la raza Holstein, lo cual se puede asociar a la disminución de la digestibilidad de la FDN, dado por la alta cantidad de carbohidratos fácilmente fermentables como el almidón (Puhakka et al., 2016). Por otro lado, la inclusión de leguminosas en las raciones de vacas de leche puede facilitar la optimización de la relación entre energía y nitrógeno fermentables, suponiendo una reducción de la síntesis de metano en el rumen y, por tanto, la emisión de este gas de efecto invernadero a la atmósfera, factor importante desde el punto de vista ambiental, debido a que se considera a los rumiantes uno de los principales contaminantes de la atmósfera (Martínez et al., 2017). También se tienen reportes de contenido de taninos condensados en niveles superiores a $56 \mathrm{~g} \mathrm{~kg}^{-1} \mathrm{MS}$ (Molina-Acaide, 2016), lo cuales, pese a ser un factor antinutricional, podrían generar la protección de la proteína para que pase a absorción en intestino, generando aumentos en el nivel de proteína en la leche (Martínez et al., 2004).

La remolacha forrajera puede incluirse hasta un $50 \%$ en la dieta de los rumiantes. Cuando se incluyó remolacha fresca en la dieta de búfalos productores de leche y se encontró un leve aumento en la producción de leche (5,9 a 61 día $^{-1}$ ) (Sorathiya et al., 2015), difiriendo en lo encontrado en este trabajo; sin embargo, coincidieron con un aumento de grasa $(6,84 \%$ a $6,99 \%)$ en la leche que se presentó con el más alto nivel de inclusión (8 $\mathrm{kg} \mathrm{vaca}^{-1}$ día $\left.^{-1}\right)$. Los 
mismos autores, relacionaron el aumento de la producción de leche con una mayor ingesta de materia seca por la alta palatabilidad y el alto contenido de agua en las raíces de remolacha.

En el trabajo realizado por Ferris et al. (2003), donde reemplazaron el $30 \%$ de ensilaje de pasto por remolacha forrajera fresca, no obtuvieron efecto significativo en la producción de leche, pero sí en el contenido de proteína en la leche, lo que difiere de este estudio, ya que la grasa fue el componente que presentó diferencia estadística $(\mathrm{p}<0,05)$ y no la proteína. Sin embargo, la diferencia en el porcentaje de proteína que se presentó en el trabajo de Ferris et al. (2003) se puede asociar más al uso del concentrado empleado como complemento a la dieta, ya que tenía un contenido de proteína de $23,4 \%$.

Los resultados obtenidos en el presente trabajo difirieron de lo encontrado por Voelker y Allen (2003), quienes evaluaron los efectos de las concentraciones crecientes $(0 \% ; 6,1 \% ; 12,1 \%$ y $24,3 \%$ de materia seca de la dieta) de pulpa de remolacha seca y peletizada, con el objetivo de reemplazar el maíz en la dieta. Obtuvieron mayores producciones $\left(36,4 ; 36,6 ; 35,9\right.$ y 35,41 día $^{-1}$ respectivamente), pero menores contenidos de grasa $(3,72 \% ; 3,84$ $\% ; 3,90 \%$ y $3,81 \%$, respectivamente) y proteína en leche $(3,21 \% ; 3,21 \% ; 3,22 \%$ y 3,10\%, respectivamente).

La cantidad de grasa y proteína de este estudio fueron similares a los obtenidos por Evans et al. (2016), quienes evaluaron cuatro niveles de inclusión de remolacha fresca $(0 \%, 8 \%, 16 \%$ y $24 \%$ del total de dieta de la materia seca), obteniendo contenidos de grasa entre 4,64 \% y 4,72\%, y de proteína entre 3,44\% y $3,54 \%$, sin diferencias estadísticas significativa entre ellos. Es de resaltar que estos autores no obtuvieron diferencia estadística significativa $(\mathrm{p}<0,05)$ en ninguno de los parámetros.

Las diferencias encontradas en la producción y calidad composicional de la leche obtenida entre este y otros estudios (Ferris et al., 2003; Voelker y Allen, 2003; Evans et al., 2016), se puede atribuir a las razas empleadas.

\section{Conclusiones}

El suministro de $8 \mathrm{~kg}$ ensilaje de haba alpargata aumentó el porcentaje de proteína en 1,4\% con respecto a no suministrar ensilaje.

El ensilaje de remolacha forrajera aumentó el porcentaje de grasa en la leche en 4,92\% frente al testigo.

El suministro de ensilaje de estas dos especies (haba alpargata y remolacha forrajera), no influyó en la producción, sólidos totales y nitrógeno ureico en leche, lo que indica que este tipo de suplementos pueden ser usados sin generar un balance energía proteína negativo.

El haba alpargata y remolacha forrajera pueden ser una alternativa de suplementación para los sistemas de producción de leche del trópico alto de Nariño durante los periodos de sequía, donde el forraje disminuye su producción hasta en $50 \%$, permitiendo disminuir el efecto de la estacionalidad y el reemplazo de fuentes comunes utilizadas para la alimentación de rumiantes.

\section{Agradecimientos}

Los autores agradecen en especial a la Corporación Colombiana de Investigación Agropecuaria (AGROSAVIA) y al macroproyecto denominado "Mejoramiento de la oferta forrajera, optimización de sistemas de alimentación

y aseguramiento de la calidad e inocuidad de la leche en el trópico alto del departamento de Nariño", ejecutado mediante recursos del Sistema General de Regalías. 


\section{Literatura citada}

AOAC (Association of Official Agricultural Chemists). 2005. AOAC Official method 972.16 milk. Mid-infrared spectroscopic method. AOAC International, MD, USA.

Anderson, V., C. Engel, M. Ostlie, J. Tebow, and D. Bernardson. 2015. Beets as feed for growing and finishing steers. North Dakota Beef Rep. 2015:4-7.

Ariza, O., O. Mayorga, B. Mojica, D. Parra, and G. Afanador. 2017. Use of LOCAL algorithm with near infrared spectroscopy in forage resources for grazing systems in Colombia. J. Near Infrared Spectrosc. 26:44-52 doi:10.1177/0967033517746900

Argamentería, A., M. Cueto, B. De-la-Roza, F. Vicente, y M. Fernández. 2005. Evaluación de la ingestión voluntaria, digestibilidad in vivo y respuesta enproducción y calidad de la leche, del ensilado de triticale/haboncillos según momento de corte. Prod. Agroganad. Gestión Efic. Conserv. Medio Nat. 1:169-174.

Arteaga, S. 2009. Elaboración de un bloque multinutricional a partir de los subproductos generados por la industria panelera, destinado para la alimentación de ganado bovino productor de carne, en el municipio de Sandoná - Nariño. Tesis Ing. Agroind. Universidad de Nariño, Nariño, COL.

Baizán, S., F. Mainar, M. González, C. González, B. De-la-Roza, A. Soldado, y A. Martínez. 2015. Alternativas forrajeras sostenibles como cultivo invernal en zonas templadas. Rev. Pastos 45(2):23-32.

Benaouda, M., A. Martínez, A. Soldado, y F. Vicente. 2013. Efecto de la asociación forrajera haba-colza como alternativa al raigrás italiano en la alimentación de vacas frisonas durante el período de transición. En: J.H. Calvo et al., editores, XV Jornadas de Producción Animal. Tomo I. Asociación Interprofesional para el Desarrollo Agrario, Zaragoza, ESP. p. 291-293.

Betancourt, J., H. Cuastumal, S. Rodríguez, J. Navia, y E. Insuasty. 2012. Alimentación de vacas holstein con suplemento de papa de desperdicio (Solanum tuberosum) y acacia negra (Acacia decurrens), y su efecto en la calidad de leche. Rev. Investig. Pecu. 1(2):41-51.

Borreani, G., A. Revello, S. Colombini, M. Odoardi, R. Paoletti, and E. Tabacco. 2009. Fermentative profiles of field pea (Pisum sativum), faba bean (Vicia faba) and white lupin (Lupinus albus) silages as affected by wilting and inoculation. Anim. Feed Sci. Technol. 151:316-323. doi:10.1016/j.anifeedsci.2009.01.020

Carulla, J., y E. Ortega. 2016. Sistemas de producción lechera en Colombia: retos y oportunidades. Arch. Latinoam. Prod. Anim. 24(2):83-87.

Checa, O. 1994. Manejo agronómico del cultivo de haba (Vicia faba). FENALCE, y CORPOICA, Pasto, COL.

Echeverry, J., L. Restrepo, y J. Parra. 2010. Evaluación comparativa de los parámetros productivos y agronómicos del pasto Kikuyo Pennisetum clandestinum bajo dos metodologías de fertilización. Rev. Lasallista Invest. 7(2):94-100.

Elgersma, A., G. Ellen, H. Horst, H. Van-Der-Boer, P. Dekker, and S. Tamminga. 2004. Quick changes in milk fat composition from cows after transition from fresh grass to a silage diet. Anim. Feed Sci. Technol. 117:13-27. doi:10.1016/j. anifeedsci.2004.08.003

Evans, E., D. Bernhardson, and J. Lamont. 2016. Case study: Effects of feeding fresh sugar beets to lactating dairy cows on milk production and milk composition. Prof. Anim. Sci. 32:253-258. doi:10.15232/pas.2015-01464

Ferris, C.P., D.C. Patterson, F.J. Gordon, and D.J. Kilpatrick. 2003. The effect of concentrate feed level on the response of lactating dairy cows to a constant proportion of fodder beet inclusion in a grass silage-based diet. Grass For. Sci. 58:1727. doi:10.1046/j.1365-2494.2003.00347.x 
Holmann, F., L. Rivas, J. Carulla, L. Giraldo, S. Guzman, M. Martínez, B. Rivera, A. Medina, y A. Farrow. 2003. Evolución de los sistemas de producción de leche en el trópico latinoamericano y su interrelación con los mercados: un análisis del caso colombiano. CIAT, COL. http://ciat-library.ciat.cgiar.org/Articulos_Ciat/tropileche/ArtCol_Esp_May_2003.pdf (consultado 16 oct. 2019).

Jefery, H. 1970. The length of change-over periods in change-desings with grazing cattle. Austr. J. Exp. Agric. Anim. Husb. 10:691-693.

Lafaux, M., J. Bastidas, y E. Insuasty. 2015. Efecto de la bovinaza en la composición nutricional del túberculo de remolacha forrajera (Beta vulgaris) en el municipio de Pasto, departamento de Nariño. Rev. Cienc. Anim. 9:209-221.

Martínez, R., N. Martínez, y M. Martínez. 2011. Diseño de experimentos en ciencias agropecuarias y biológicas con SAS, SPSS, R y STATISTIX. Fondo Nacional Universitario, Bogotá, COL.

Martínez, T.F., F.J. Moyano, M. Diaz, F.G. Barroso, and F.J. Alarcón. 2004. Ruminal degradation of tannin-treated legume meals. J. Sci. Food Agric. 84:1979-1987. doi:10.1002/jsfa.1907

Martínez, A., F. Vicente, S. Baizán, y N. Barhoumi. 2017. Interés agronómico de la inclusión de las habas forrajeras en las raciones de rumiantes en la Cornisa Cantábrica. Afriga 131:88-96.

Molano, R., F. Aguilar, J. Carulla, y G. Afanador. 2011. Sistemas integrados de alimentación en bovinos. Universidad Nacional de Colombia, Bogotá, COL.

Molina-Alcaide, E. 2016. Las leguminosas en nutrición de ruminates. En: XXVIII Foro de Colaboración Público-Privada "Leguminosas. Producción y consumo. Una revolución pendiente". INIA, ESP. http://wwwsp.inia.es/Investigacion/ OtrasUni/TransferenciaTecnologia/ForosINIA/Legumin/Lists/Presentaciones/Attachments/28/27EduardaMolinaEEZ.pdf (consultado 15 mar. 2016).

Montgomerie, W. 2005. Ten years experience of multi-breed evaluations and crossbreeding in New Zealand. Proc. Interbull. Meetin 33:98-101.

Morales, A., J. León, E. Cárdenas, G. Afanador, y J. Carulla. 2013. Composición química de la leche, digestibilidad in vitro de la materia seca y producción en vacas alimentadas con grampíneas solas o asociadas con Lotus uliginosus. Rev. Med. Vet. Zoot. 60(1):32-48

Mordenti, A., F. Merendi, M. Fustini, and A. Formigoni. 2007. Effects of different protein plants in cows diet on milk for Parmigiano Reggiano production. Ital. J. Anim. Sci. 6:463-465. doi:10.4081/ijas.2007.1s.463

Osmane, B., I. Konosonoka, A. Trupa, and L. Proskina. 2017. Peas and beans as a protein feed for dairy cows. Agron. Res. 15:2026-2038. doi:10.15159/AR.17.027

Puhakka, A.L., S. Jaakkola, I. Simpura, T. Kokkonen, and A. Vanhatalo. 2016. Effects of replacing rapeseed meal with faba bean at 2 concentrate crude protein levels on feed intake, nutrient digestion, and milk production in cows fed grass silage - based diets. J. Dairy Sci. 99:17993-8006. doi:10.3168/jds.2016-10925

Rodríguez, R., A. Sosa, y Y. Rodríguez. 2007. La síntesis de proteína microbiana en el rumen y su importancia para los rumiantes. Rev. Cub. Cienc. Agríc. 41(4):303-311.

Rowarth, J., 2013. Dairy cows - Economic production and environmental protection. In: J. Dymond, editor, Ecosystem services in New Zealand - conditions and trends. Manaaki Whenua Press, Lincoln, NZL. p. 85-93.

Sañudo, B., O. Checa, y G. Arteaga. 1999. Manejo agronómico de leguminosas en zona crealistas. PRODUMEDIOS, Pasto, COL. 
Sorathiya, L.M., M.D. Patel, K.K. Tyagi, A.B. Fulsoundar, and A.P. Raval. 2015. Effect of sugar beet tubers as a partial replacer to green fodder on production performance and economics of lactating Surti buffaloes in lean period. Vet. Word 8:15-18. doi:10.14202/vetworld.2015.15-18

Stobbs, T., and R. Sandland. 1971. The use of a latin square change-over design with dairy cows to detect differences in the quality of tropical pastures. Austr. J. Exp. Agric. Anim. Husb. 12:463-469.

Trujillo, G. 2009. Guía para la utilización de recursos forrajeros tropicales para la alimentación de bovinos. Convenio 0367/09. Comité de Ganaderos del Huila, SENA, Fondo Granadero del Huila, y Gobernación del Huila, Huila, COL.

Voelker, J., and M. Allen. 2003. Pelleted beet pulp substituted for high-moisture corn: 1. Effects on feed intake, chewing behavior, and milk production of lactating dairy cows. J. Dairy Sci. 86:3542-3552. doi:10.3168/jds.S0022-0302(03)73959-9

Volpelli, L.A., M. Comellini, M. Gozzi, F. Masoero, and M. Moschini. 2012. Pea (Pisum sativum) and faba beans (Vicia faba) in dairy cow diet: Effect on milk production and quality. Ital. J. Anim. Sci. 11:217-222. doi:10.4081/ijas.2012.e40

Volpelli, L.A, M. Comellini, F. Masoero, M. Moschini, D.P. Lo-Fiego, and R. Scipioni. 2009. Pea (Pisum sativum) in dairy cow diet: effect on milk production and quality. Ital. J. Anim. Sci. 8:245-257. doi:10.4081/ijas.2009.245

Volpelli, L.A., M. Comellini, F. Masoero, M. Moschini, D.P. Lo-Fiego, and R. Scipioni. 2010. Faba bean in dairy cow diet: Effect on milk production and quality. Ital. J. Anim. Sci. 9:138-144. doi:10.4081/ijas.2010.e27 